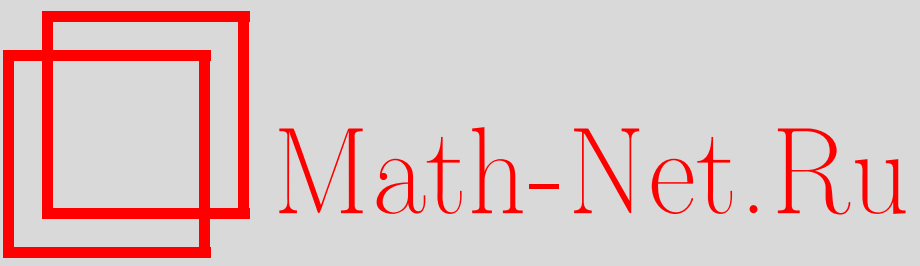

И. М. Кричевер, С. П. Новиков, Голоморфные расслоения и коммутирующие разностные операторы. Двухточечные конструкции, УМН, 2000, том 55, выпуск $3,181-182$

DOI: https://doi.org/10.4213/rm302

Использование Общероссийского математического портала Math-Net.Ru подразумевает, что вы прочитали и согласны с пользовательским соглашением

http: //www . mathnet.ru/rus/agreement

Параметры загрузки:

IP: 34.229 .45 .116

26 апреля 2023 г., 17:43:48 


\title{
ГОЛОМОРФНЫЕ РАССЛОЕНИЯ И КОММУТИРУЮШИЕ РАЗНОСТНЫЕ ОПЕРАТОРЫ. ДВУХТОЧЕЧНЫЕ КОНСТРУКЦИИ
}

\author{
И. М. КРИЧЕвЕР, С.П. НовиКов
}

Настоящая работа непосредственным примыкает к предшествующей работе авторов [1]. Как известно, в современной математической физике теория коммутирующих одномерных операторов появилясь, как побочный алгебраический аспект теории иинтегрирования нелинейных солитонных систем и спектральной теории периодических конечнозонных операторов [2]-[5].

Как чисто алгебраическая задача, проблема классификации обыкновенных скалярных дифференциальных операторов была поставлена еще в 20-х годах Бурхналом и Чаунди [6], которые далеко продвинулись в решении задачи для случая операторов взаимно-простых порядков (в котором ранг всегда равен 1), завершенном в [3]. Они же отмечали, что общая задача для ранга $r>1$, представляется чрезвьгайно трудной.

Первые шаги были сделаны в работах [7], [8]. Метод эффективной классификации коммутирующих дифференциальных операторов ранга $r>1$ общего положения был создан авторами в работах [9], [10]. Коммутирующие пары ранга $r$ зависят от $(r-1)$ произвольной функции одной переменной, гладкой алгебраической кривой $\Gamma$ с одной отмеченной точкой $P$ и набора параметров Тюрина (характеризующих оснащенное стабильное голоморфоное расслоение). Мы называем такие конструкции одноточечным.ми.

Для разностных операторов вся, ставшая уже классической, теория пар коммутирующих операторов ранга $r=1$ основывалась только на двухточечных конструкциях [11], [12]. Кольца таких операторов оказывались изморфны кольцам $A\left(\Gamma, P^{ \pm}\right)$мероморфныт функций на алгебраической кривой Г с полюсами в паре отмеченных точек $P^{ \pm}$.

В предшествуюшей работе авторов [1] было показано, что для ранга $2 l \geqslant 2$ широкий класс коммутирующих разностных операторов получается из одноточечной конструкции. Аналогично непрерывному случаю, эти операторы зависят от произвольных функции одной переменной $n \in Z$.

$\mathrm{B}$ настоящей работе нами получено описание широкого класса коммутирующих разностных операторов, построенных исходя из двухточечных конструкции. В отличие от одноточечных конструкции, здесь не возникает никаких произвольных функции; коэффициенты операторов могут быть вычислены через тэта-функции Римана. Как и для случая ранга 1, эти операторы приводят к решениям уравнений $2 D$ цепочки Тода и всей связанной с ними иерархии.

Рассмотрим гладкую алгебраическую кривую Г рода $g$ с двумя отмеченными точками $P^{ \pm}$. Пусть $(\gamma)$ является набором $r g$ точек $\gamma_{s}, s=1, \ldots, r g$, на $\Gamma$, а $(\alpha)$ является набором $(r-1)$ мерных векторов: $\alpha_{s}=\left(\alpha_{s j}\right), j=1, \ldots, r-1$. Согласно [9], [10], эти параметры $(\gamma, \alpha)$, назьваются параметрами Тюрина. В общем случае они определяют стабильное оснащенное расслоение $\mathscr{E}$ над $Г$ ранга $r$ и степени $c_{1}(\operatorname{det} \mathscr{E})=r g$.

ЛЕмма 1. Для любого набора параметров Тюрина $(\gamma, \alpha)$ общего положсения существует единственная мероморфная вектор-функция $\psi_{n}(Q)=\left(\psi_{n}^{i}(Q)\right), i=1, \ldots, r ; Q \in \Gamma$ такал, что: (i) вне $P^{ \pm}$функция $\psi_{n}^{i}(Q)$ имеет не более чем простые полюсы в точках $\gamma_{s}$; ее вычеты в әтих точках удовлетворяют соотношению $\alpha_{s i} \operatorname{res} \gamma_{s} \psi_{n}^{i}=\operatorname{res} \gamma_{s} \psi_{n}^{r}$; (ii) в окрестности отмеченных точек $\psi_{n}(Q)^{i}, n=k r+j, 0 \leqslant j<r$, имеет вид

$$
\psi_{n}^{i}=z_{ \pm}^{\mp k}\left(\xi_{n, \pm}^{i}+O\left(z_{ \pm}\right)\right)
$$

əде $\xi_{k r+j,+}^{j}=1, \xi_{k r+j,+}^{i}=0, i>j ; \xi_{k r+j,-}^{i}=0, i<j$ (здесь $z_{ \pm}$локальнье координать в окрестностях $\left.P^{ \pm}\right)$.

Определенная выше вектор-функция является аналогом разностной функции Бейкера-Ахиезера для случая высших рангов.

Работа выполнена при частичной поддержке грантов DMS-98-02577, DMS-97-04613. 
Теорема 1. Пусть $\psi_{n}^{i}(Q)$ является векторной функцией Бейкера-Ахиезера, соответствующей набору алгебро-геометрических данных $\left\{\Gamma, P^{ \pm},(\gamma, \alpha)\right\}$. Тогда для любой функции $f(Q) \in A\left(\Gamma, P^{ \pm}\right)$существует единственньй разностный оператор $L_{f}$ вида:

$$
L_{f}=\sum_{i=-r n_{-}}^{r n_{+}} u_{i}(n) T^{i}, \quad u_{ \pm r n_{ \pm}}=f_{ \pm} \neq 0
$$

mакой, что $L_{f} \psi_{n}^{i}(Q)=f(Q) \psi_{n}^{i}(Q), i=1, \ldots, r$. Здесь $T y_{n}=y_{n+1}$ оператор сдвига, $а$ $n_{ \pm}$поряджи функции $f(Q)$ в точках $P^{ \pm}$.

Как функчия $\psi$, так и коэффициенты операторов могут быть явно вычислены через тәта-функцию Римана, отвечающ, кривой $\Gamma$. Коэффициенты операторов $L_{f}$ являются периодическими функциями тогда и только тогда, когда $\mathrm{A}\left(P^{+}\right)-A\left(P^{-}\right)$является точкой конечного порядка на якобиане $J(\Gamma)$.

ЗАмечАниЕ 1. Обратим внимание на то, что в этой конструкции нет функциональных параметров даже и в случае ранга $r>1$. Из формула $(2)$ следует, что среди операторов $L_{f}$ имеются как такие, что все сдвиги $T^{i}$ положительны, $i>0$, так и такие операторы, что все сдвиги отрицательны, $i<0$. Одноточечные конструкции никогда не приводят к таким операторам. Мы естествено приходим здесь и к конструкции представлений варианта алгебры Каца-Муди $\widehat{s l}(r, C)$, ассоциированной с алгебраической кривой $\Gamma$ с отмеченными точками $P^{ \pm}$по схеме работы [13].

ЗАмечАниЕ 2. Авторы также построили для любого ранга $r>1$ двухточечные вектор-функции Бейкера-Ахиезера, задающие решения полной иерархии уравнений $2 D$ цепочки Тода. Они зависят от $2(r-1)$ произвольных функций. Эта конструкция содержит теорию интегрируемых потенциалов двумерного оператора Шредингера, связанных с раслоениями высших рангов, начатую авторами в работе [14].

Рассмотрим кольцо $\mathscr{D}$ разностных операторов конечного порядка. В силу теоремы 1 набор параметров Тюрина общего положения $(\gamma, \alpha)$ задает гомоморфизм $G_{(\gamma, \alpha)}: A\left(\Gamma, P^{ \pm}\right) \mapsto \mathscr{D}$, образ которого является максимальным коммутативным подкольцом. Следующая теорема показывает, что предложенная конструкция описывает все подобные кольца общего положения.

Теорема 2. Для любого кольцевого мономорфизма $G: A\left(\Gamma, P^{ \pm}\right) \mapsto \mathscr{D}$, такого что операторы $L_{f}=G(f)$ имеют вид (2), нормированные совместные собственные функиии определяют оснащенное голоморфное расслоение ранга $r$ и степени $\mathrm{rg}$. $B$ общем положсении, когда это расслоение описывается параметрами Тюрина $(\gamma, \alpha)$, гомоморфизм $G$ совпадает с гомоморфизмом $G=G_{(\gamma, \alpha)}$, задаваемым в силу теореми 1 с помощью соответствующей векторной функции Бейкера-Ахиезера.

\section{СПИСОК ЛИТЕРАТУРЫ}

[1] Кричевер И. М., Новиков С. П. // УМН. 2000. Т. 55. №1. С. 187-188. [2] Дубровин Б.А., Матвеев В.Б., Новиков С.П. // УМН. 1976. Т. 31. № 1. С. 55-136. [3] Кричевер И. М. // УМН. 1977. Т. 32. №6. С. 183-208. [4] Дубровин Б. А., Кричевер И. М., Новиков С. П. Итоги науки и техники. Фундаментальные проблемы математики. М.: ВИНИТИ, 1985. [5] Захаров В.Е., Манаков С. В., Новиков С. П., Питаевский Л. П. Теория солитонов. Метод обратной задачи. М.: Наука, 1980. [6] Burchnall J. L., Chaundy T.W. // Proc. Roy. Soc. London. 1928. V. 118. P. 557-583. [7] Dixmier J. // Matematika. 1969. V. 13. № 4. P. 27-40. [8] Дринфельд В. Г. // Функц. анализ и его прил. 1977. Т. 11. № 1. С. 11-31. [9] Кричевер И. М. // Функц. анализ и его прил. 1978. Т. 12 . № 3. С. 20-31. [10] Кричевер И. М., Новиков С. П. // Функц. анализ и его прил. 1978. Т. 12. № 4. С. 41-52. [11] Кричевер И. М. // УМН. 1978. Т. 33. № 4. С. 215-216. [12] Mumford D. // Proccedings of International Symposium on Algebraic Geometry (Kyoto Univ., Kyoto 1977). Tokyo: Kinokuniya Book Store, 1978. Р. 115-153. [13] Кричевер И. М., Новиков С. П. // Функц. анализ и его прил. 1987. Т. 21. № 2. С. 46-63. [14] Кричевер И. М., Новиков С. П. // УМH. 1980. T. 35. №6. С. 47-68.

Columbia University, New York, University of Maryland, College Park, Принято редколлегией Институт теоретической физики им. Ландау 